\title{
Evolutionary genetics: 150 years of natural selection
}

\author{
Preface
}

This year marks a hundred and fifty years since the formal enunciation of the principle of natural selection as a driver of adaptive evolution, through the reading of papers by Charles Darwin and Alfred Wallace to the Linnaean Society at London. It is also a hundred years since the independent publication of papers by Wilhelm Weinberg and G. H. Hardy that together laid the foundations of population genetics. If that were not enough to make this an important anniversary for evolutionary genetics, it is also ninety years since the publication of R. A. Fisher's landmark paper reconciling the statistical results of the biometricians with the discrete form of inheritance explained by Mendelian principles, creating the field of quantitative genetics and making possible the interface between genetics and evolution that led eventually to the Neo-Darwinian Synthesis in the mid-twentieth century.

Since Darwin's early speculations on mechanisms of particulate inheritance, genetics has been very closely intertwined with evolution, for after all it is heredity that provides the link between generations of discrete organisms whose changing characteristics over generations are explained by evolutionary theory. The Journal of Genetics has been active in publishing research on the interface between genetics and evolution practically since its inception in 1910, and especially during the decades of J. B. S. Haldane's stewardship of the journal. We are therefore very pleased to mark the various anniversaries referred to above with this special issue on 'Evolutionary genetics: 150 years of natural selection'. The eleven articles in this issue are intended to provide a combination of some historical overview on important questions in evolutionary genetics and vignettes of present day research in the field. The coverage is not comprehensive, and there are some emerging areas that, unfortunately, could not be covered.

Ayala's lead article puts Darwin's contribution to our intellectual and scientific world-view into historical perspective, discussing the importance of his thesis in bringing the living world into the domain of rationally explainable natural phenomena. Kulathinal and Singh review advances in our understanding of the molecular genetic mechanisms underlying the fundamental evolutionary process of speciation that is ultimately responsible for the stunning diversity of life-forms. Roff takes up a fundamental concept in evolutionary theory-Darwinian fitness - and discusses the pros and cons of a number of formulations of fitness in different kinds of evolutionary genetic models. Crow's piece suggests several mechanisms for how additive genetic variation for fitness-related traits is maintained in populations, thus retaining evolvability, a concept that has been much studied in recent years. Fry discusses the use of the model organism Drosophila melanogaster to experimentally assess genotype-by-environment interaction for total fitness, an issue of some importance given the view that environmental heterogeneity promotes the maintenance of genetic variation through such interactions.

The study of morphology, along with comparative anatomy, played an important role in the development of evolutionary thinking in the decades before and after Darwin. Today, morphometrics is again an exciting field, with ever more sophisticated methods being used to understand the evolution of form. Chakir et al. report a study aimed at making a start in unravelling the underlying quantitative genetics of various morphometric traits in African and European populations of $D$. melanogaster. Debat et al. address the issue of how stressful environments might affect the evolution of morphometric characters by examining phenotypic variation in wing shape at different temperatures in populations of $D$. melanogaster from different environments in the same geographical location. Their study also looks at fluctuating asymmetry and its relationship with individual variation, and their results suggest that the developmental processes underlying individual variation and fluctuating asymmetry might not be identical. While we do not review the field of evolutionary development biology, it is not because we consider it unimportant. Hopefully, this topic will be explored in later issues of the Journal of Genetics.

Ageing is a topic of perennial interest to geneticists and non-geneticists alike. In their crisp review, Rose et al. outline the key theoretical and experimental milestones in the development of our understanding of why and how ageing evolves. They also highlight the importance of an evolutionary perspective not just for our understanding of ageing but also for our attempts at intervention in the process of human ageing. 
In general, one of the important applications of evolutionary thinking that has emerged over the past fifteen years or so is the area often called Darwinian medicine or evolutionary medicine, a belated recognition of the fact that an evolutionary perspective can be very helpful in how we think about disease and its prevention, cure or management.

Sexual dimorphism for traits not directly related to reproduction is common in multicellular animals, and is one way of evolutionarily circumventing inter-sexual conflict arising due to different selection pressures on males and females. Two papers in this issue address the broad question of differential selection on the sexes and how it may influence the evolution of sexual dimorphism. Zwaan et al. selected differentially for male and female development time in the butterfly Bicyclus anynana, and report that a strong between-sex correlation constrains the independent evolutionary fine-tuning of male and female development time in this species. They also find that the degree of sexual dimorphism in development time is quite sensitive to rearing temperature. Kwan et al. report on a study exploring interactions between intra-locus sexual conflict, sexual dimorphism, and environmental variation by examining male and female evolutionary responses to desiccation stress in D. melanogaster. Their results suggest that some traits, such as size, changed in a sex-specific manner as the populations adapted to the desiccation stress, but that the tolerance to desiccation appeared to be genetically correlated between sexes. This study also exemplifies the strengths of experimental evolution in teasing out various factors and trade-offs, leading to a deeper understanding the evolution of complex phenotypes.

In the last, and in the scope of its purview perhaps the broadest, paper in this issue, Grafen reviews the ongoing Formal Darwinism Project that aims at reconciling the population genetics view on evolution with the notion that evolution via natural selection tends to optimize fitness. This is a fundamental issue in evolutionary biology, especially because many of the extensions of evolutionary thinking to animal and human behaviour and sociality are implicitly founded on an optimization argument. There have been some earlier attempts at harmonizing the optimization and population genetic approaches to evolution, but the results have not been very convincing. If this ongoing project succeeds in reconciling these two approaches, it will indeed be a useful contribution to the refinement of evolutionary understanding.

Overall, the articles in this issue provide an overview, albeit patchy, of how far our appreciation and understanding of the subtlety of the process of adaptive evolution through natural selection have come in the past hundred and fifty years. Nevertheless, some of the articles also underscore how much is still left to understand about the manner in which ecology, genetics and development interact to produce the diversity in and of life-forms that is perhaps the most striking characteristic feature of the living world. There are enough interesting questions in evolution yet to engage several more generations of evolutionary biologists in challenging and enjoyable research; we certainly look forward to such continuing enjoyment. Finally, it is a pleasure to thank all the contributors to this special issue, as well as all those who generously spared the time to review the articles.

Амitabh Joshi
Laurence D. Mueller
Editors

E-mail: ajoshi@jncasr.ac.in; ldmuelle@uci.edu. 